\title{
Measurement of the separation between atoms beyond diffraction limit
}

\author{
Jun-Tao Chang, ${ }^{1}$ Jörg Evers, ${ }^{1,2}$ Marlan O. Scully, ${ }^{1,3}$ and M. Suhail Zubairy ${ }^{1, *}$ \\ ${ }^{1}$ Institute for Quantum Studies and Department of Physics, Texas A\&M University, College Station, Texas 77843-4242, USA \\ ${ }^{2}$ Max-Planck-Institut für Kernphysik, Saupfercheckweg 1, D-69117 Heidelberg, Germany \\ ${ }^{3}$ Princeton Institute for Materials Research, Princeton University, Princeton, NJ 08544-1009, USA
}

(Received 6 June 2005; published 10 March 2006)

\begin{abstract}
Precision measurement of small separations between two atoms or molecules has been of interest since the early days of science. Here, we discuss a scheme which yields spatial information on a system of two identical atoms placed in a standing wave laser field. The information is extracted from the collective resonance fluorescence spectrum, relying entirely on far-field imaging techniques. Both the interatomic separation and the positions of the two particles can be measured with fractional-wavelength precision over a wide range of distances from about $\lambda / 550$ to $\lambda / 2$.
\end{abstract}

DOI: 10.1103/PhysRevA.73.031803

PACS number(s): 42.50.Ct, 42.30.- d, 42.50.Fx

The measurement of small distances is a fundamental problem since the early days of science. It has become even more important due to recent interest in nanoscopic and mesoscopic phenomena [1]. Starting from the invention of the optical microscope around 400 years ago, today's optical microscopy methodologies can basically be divided into lensbased and lensless imaging. In general, far-field imaging is lens-based and thus limited by criteria, such as the Rayleigh diffraction limit which states that the achievable resolution in the focus plane is limited to half of the wavelength of illuminating light. Further limitation arises from out-of-focus light, which affects the resolution in the direction perpendicular to the focal plane. Many methods have been suggested to break these limits [2-8]. Lens-based techniques include confocal, nonlinear femtosecond, or stimulated emission depletion microscopy [3]. Also nonclassical features, such as entanglement, quantum interferometry, or multiphoton processes, can be used to enhance resolution [4-6]. A particularly promising development is lensless near-field optics, which can achieve nanometer spatial resolution [2]. Roughly speaking, the idea is to have light interactions close enough to the object to avoid diffraction. This, however, typically restricts near-field optics to objects on a surface. In 1995, Betzig proposed a method to reach subwavelength resolution that is not limited to one spatial dimension by assuming nonidentical, individually addressable objects [7]. Subsequently, this was realized in a landmark experiment of Hettich et al. [8]. It combined near-field and far-field fluorescence spectroscopy techniques, using the fluorescence spectrum to label different molecules inside an inhomogeneous external electrical field. They also noticed dipoledipole interactions between adjacent objects [9-11] and used it to correct the measurement result. However, there is still great interest in achieving nanometer distance measurements by using optical illuminating far-field imaging only.

In this communication, we propose a scheme to measure the distance between two adjacent two-level systems by driving them with a standing wave laser field and measuring the

\footnotetext{
*Electronic address: zubairy@physics.tamu.edu
}

far-field resonance fluorescence spectrum, which is motivated by the localization of single atom inside a standing wave field [12,13]. In particular, we focus on distances smaller than the Rayleigh limit $\lambda / 2$. Our basic approach is that in a standing wave, the effective driving field strength depends on the position of the particles. Thus, each particle generates a sharp sideband peak in the spectrum, where the peak position directly relates to the subwavelength position of the particle. As long as the two sideband peaks can be distinguished from each other, the position of each particle can be recovered. However, when the interatomic distance decreases, the two particles can no longer be considered independent. Due to the increasing dipole-dipole interaction between the two particles, the fluorescence spectrum becomes complicated. We find, however, that the dipole-dipole interaction energy can directly be extracted from the fluorescence spectrum by adjusting the parameters of the driving field. Since the dipole-dipole interaction energy is distance dependent, it yields the desired distance information. We provide detailed measurement procedures and our estimates show that the scheme is applicable to inter-particle distances in a very wide range from $\lambda / 2$ to about $\lambda / 550$.

Our model system consists of two identical two-level atoms located at fixed points $\mathbf{r}_{i}=\left(x_{i}, y_{i}, z_{i}\right)^{T}(i=1,2)$ in a resonant standing wave laser field (see Fig. 1). The atomic transition frequency is $\omega_{0}$. The laser field has frequency $\omega_{L}$, wavelength $\lambda$ and wave vector $\mathbf{k}=k \hat{\mathbf{z}}$. We assume the two atoms to be arranged along $\hat{\mathbf{z}}$. The driving field Rabi fre-

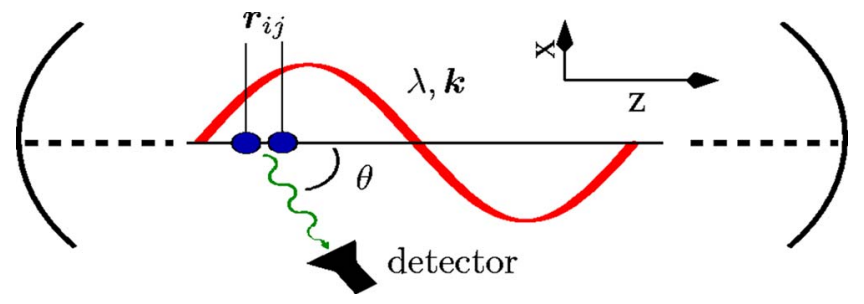

FIG. 1. (Color online) Two atoms in a standing wave field separated by a distance $\left|\mathbf{r}_{i j}\right|$ smaller than half of the wavelength $\lambda$ of the driving field. The distance of the two atoms is measured via the emitted resonance fluorescence. 
quency of atom $i$ is $\Omega_{i}$, with $\Omega_{i}=\Omega \sin \left(k \cdot z_{i}\right)$. We denote the raising (lowering) operator of the $i$ th atom by $S_{i}^{+}\left(S_{i}^{-}\right)$. In the following, we assume the transition dipole moments of the two atoms to be parallel and aligned perpendicular to the $\hat{\mathbf{z}}$ direction. We also assume resonant driving, $\Delta=\omega_{L}-\omega_{0}=0$.

If the two atoms are far apart (the distance between atom $i$ and $j\left|z_{i j}\right|=\left|z_{i}-z_{j}\right| \gg \lambda$ ), then they are independent, and the total Master equation is given by the sum of the two singleparticle Master equations [10]. If the two atoms come close, they dipole-dipole interact, causing a collective system dynamics. This gives rise to a complex energy shift due to a virtual photon exchange between the two atoms. The imaginary part of this shift corresponds to an incoherent coupling, whereas the real part shifts the energy of the collective states of the system. The full collective Master equation is given by $[9,10]$

$$
\frac{\partial \rho}{\partial t}=\frac{1}{i \hbar}[H, \rho]-\sum_{i, j=1}^{2} \gamma_{i j}\left(\left[S_{i}^{+}, S_{j}^{-} \rho\right]-\left[S_{j}^{-}, \rho S_{i}^{+}\right]\right) .
$$

The coherent evolution is governed by $H=H_{0}+H_{d d}+H_{L}$. The free energy $H_{0}=(\hbar / 2) \omega_{0} \Sigma_{i=1}^{2}\left(S_{i}^{+} S_{i}^{-}-S_{i}^{-} S_{i}^{+}\right)$of the two atoms and the interaction with the driving laser field $H_{L}$ $=(\hbar / 2) \sum_{i=1}^{2}\left(\Omega_{i} S_{i}^{+} e^{-i \omega_{L} t}+\right.$ H.c. $)$ are the same as for two independent atoms. The coherent energy shift of the collective states arises from the dipole-dipole interaction $H_{d d}$ $=\hbar \Omega_{12}\left(S_{1}^{+} S_{2}^{-}+\right.$H.c. $)$, which involves couplings of both atoms. For the considered geometry, the dipole-dipole interaction $\Omega_{12}$ is given by

$$
\Omega_{12}=\frac{3}{2} \gamma\left\{-\frac{\cos \left(k z_{i j}\right)}{\left(k z_{i j}\right)}+\frac{\sin \left(k z_{i j}\right)}{\left(k z_{i j}\right)^{2}}+\frac{\cos \left(k z_{i j}\right)}{\left(k z_{i j}\right)^{3}}\right\} .
$$

The incoherent evolution first entails the independent spontaneous emission of the two atoms $\gamma_{i i}(i=1,2)$, as found for uncoupled atoms. Terms with $\gamma_{i j}(i \neq j)$ are the incoherent dipole-dipole couplings, where

$$
\gamma_{i j}=\frac{3}{2} \gamma\left\{\frac{\sin \left(k z_{i j}\right)}{\left(k z_{i j}\right)}+\frac{\cos \left(k z_{i j}\right)}{\left(k z_{i j}\right)^{2}}-\frac{\sin \left(k z_{i j}\right)}{\left(k z_{i j}\right)^{3}}\right\} .
$$

For large distances, $\left(k z_{i j} \gg 1\right)$, we find $\Omega_{12} \approx 0$ and $\gamma_{i j}$ $\approx \gamma \delta_{i j}$, where $\delta_{i j}$ is the Kronecker Delta symbol. Thus we recover the case of two independent atoms, as expected. For small distances $\left(k z_{i j} \ll 1\right)$, one finds maximum incoherent cross-coupling, and $\Omega_{12}$ approaches the static dipole-dipole interaction,

$$
\Omega_{12} \approx 3 \gamma /\left[2\left(k z_{i j}\right)^{3}\right], \quad \gamma_{i j} \approx \gamma .
$$

Our strategy is to identify the distance of the two atoms via the emitted resonance fluorescence. We define $\hat{\mathbf{R}}$ as the unit vector in observation direction, and the observation angle $\theta$ as $\theta=\operatorname{arcos}\left(\hat{\mathbf{R}} \cdot \mathbf{r}_{12} / r_{12}\right)$. The total two-atom steady state resonance fluorescence spectrum $S(\omega)$ up to a geometrical factor is given by [10]
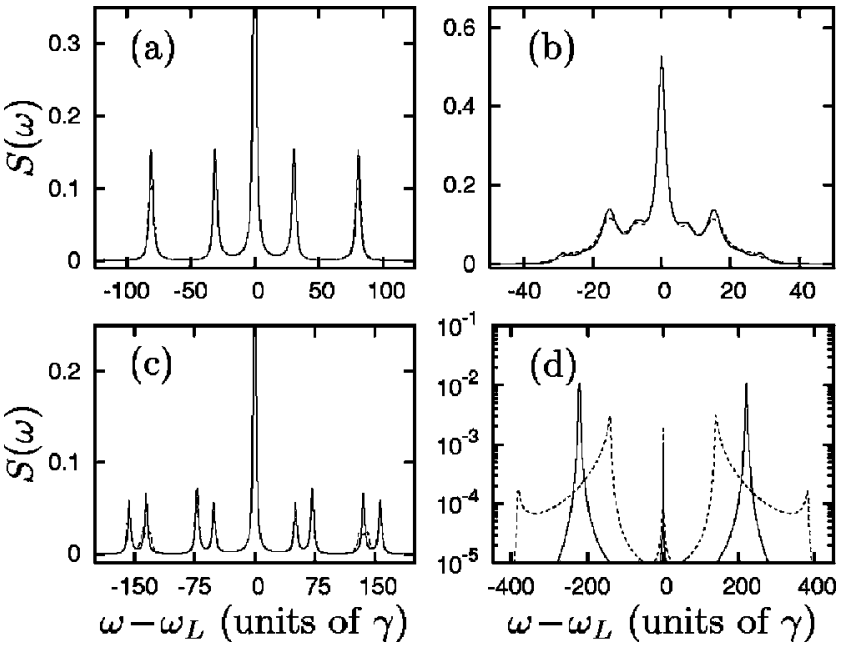

FIG. 2. Sample spectra for $\Delta=0, \theta=\pi / 2, z_{1}=0.05 \lambda$. Fixed distance $z_{12}$ (solid lines) and harmonic oscillation around $z_{12}$ (dashed lines). (a) Large separation case: $z_{12}=0.3 \lambda, \Omega=100 \gamma$. (b) Intermediate separation, weak driving field: $z_{12}=0.08 \lambda, \Omega=20 \gamma$ (c) As (b), but strong driving field: $z_{12}=0.08 \lambda, \Omega=200 \gamma(\mathrm{d})$ Small separation: $z_{12}=0.03 \lambda, \Omega=20 \gamma$.

$$
S(\omega)=\operatorname{Re} \int_{0}^{\infty} d \tau e^{i\left(\omega-\omega_{L}\right) \tau} \sum_{i, j=1}^{2}\left\langle S_{i}^{+}(0) S_{j}^{-}(\tau)\right\rangle_{s} e^{i k \hat{\mathbf{R}} \cdot \mathbf{r}_{i j}}
$$

where the subindex $s$ denotes the steady state. In general, this resonance fluorescence spectrum is rather complicated [11]. The spectrum, however, simplifies considerably in limiting cases, where either the driving field Rabi frequency or the dipole-dipole interaction dominates the dynamics. This will be exploited in the following, where we present in detail a measurement procedure, which allows us to extract the distance between the two atoms and their positions relative to nodes of the standing wave field, both with fractionalwavelength precision. The first step in the measurement sequence is to apply a standing wave laser field to the two atoms, which at an antinode of the standing wave corresponds to a Rabi frequency $\Omega$ of a few $\gamma$. Depending on the relative separation of the atoms, different spectra can be observed.

If the two atoms are well-separated (about $\lambda / 10 \leqq z_{12}$ $\leq \lambda / 2$ ), then the dipole-dipole interaction is negligible. In this case, spectra as shown in Fig. 2(a) are obtained. The two sideband structures can be interpreted as arising from the ac-Stark splitting due to $\Omega_{1}$ and $\Omega_{2}$. Thus, the sideband peak positions $\nu_{1}^{p}$ and $\nu_{2}^{p}$ can directly be related to $\Omega_{1}$ and $\Omega_{2}$ and therefore to the position of the two atoms relative to the standing wave field nodes. Consequently, we can obtain the distance $z_{12}$. Within half a wavelength, however, in general two interatomic distances are possible for measured values of $\Omega_{1}$ and $\Omega_{2}$ [see Figs. 3(a) and 3(b)] [14]. An identification of the actual atomic positions is possible by changing the standing wave phase slightly, i.e., shifting the positions of the (anti-) nodes. As shown in Figs. 3(a) and 3(b), a combination of the possible positions for two different standing wave phases yields the actual separation. Note that this complica- 

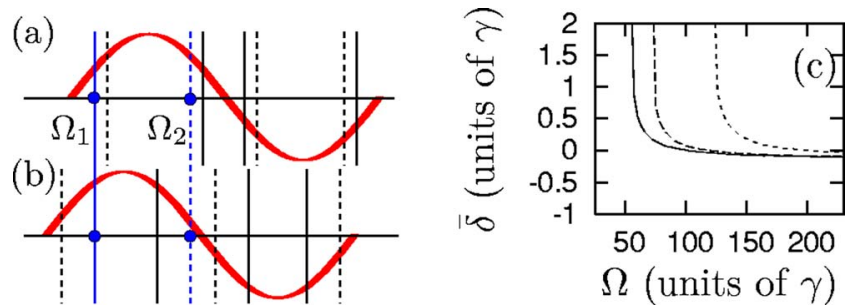

FIG. 3. (Color online) (a,b) Obtaining the position of the two atoms via a phase shift of the standing wave field. Solid (dashed) lines show possible atom positions for given $\Omega_{1}\left(\Omega_{2}\right)$. (a) Before, (b) after the phase shift. The only coinciding potential positions in (a) and (b) give the true atomic positions. (c) Deviation $\bar{\delta}$ $=\sigma_{\mathrm{p}}-2 \Omega_{12}$ of the doublet splitting $\sigma_{\mathrm{p}}$ from $2 \Omega_{12}$ for the strong field, intermediate distance case. $z_{12}=0.08 \lambda, \theta=\pi / 2$, and $\Delta=0$. The positions of the atoms are $z_{1}=0.05 \lambda$ (solid), $0.1 \lambda$ (dashed), $0.15 \lambda$ (dotted).

tion is not present for nearby atoms, where the nonvanishing dipole-dipole energy allows to determine the distance directly. In Fig. 2(a), the distance of the two particles is $z_{12}$ $=0.3 \lambda$. From the spectrum accessible in experiments, the distance $z_{12}^{\text {expt }}=(0.300 \pm 0.02) \lambda$ is obtained, if we allow for a total measurement uncertainty of about $10 \%$. Thus, the actual and the measured distances match, and the $10 \%$ uncertainty of the distance measurement corresponds to about $\lambda / 50$.

If the distance between the two atoms is intermediate (about $\lambda / 30 \leqq z_{12} \lesssim \lambda / 10$ ), then the initial weak-field measurement in general yields a more complicated spectrum, see Fig. 2(b). The reason is that then the dipole-dipole coupling and the driving field strength are comparable, and the two atoms are not independent. In such a case, a quantitative interpretation of the spectrum is difficult. However, increasing the Rabi frequency $\Omega$ leads to a spectrum as shown in Fig. 2(c). The spectrum consists of a central peak, two inner sideband doublets, and two outer sideband doublets, each symmetrically placed around the driving field frequency $\omega_{L}$. The center positions of the inner and outer sideband doublets corresponds to the Rabi frequencies $\Omega_{1}$ and $\Omega_{2}$. The sideband structures are split into doublets due to the dipoledipole coupling of the two atoms. For large $\Omega$, the splitting approaches twice the energy $\Omega_{12}$, as shown in Fig. 3(c). Thus the strong-field sideband doublet splitting directly yields $\Omega_{12}$ and then the distance of the two atoms, via Eqs. (2). For example, in Fig. 2(c), the actual distance is $z_{12}=0.08 \lambda$. From the spectrum, a measurement would obtain $\Omega_{12}$ $=(10.54 \pm 1.05) \gamma$, where again we have allowed for an uncertainty of about $10 \%$. From Eq. (2), this yields a measured distance of $z_{12}=(0.0801 \pm 0.0027) \lambda$, in good agreement with the actual value. On the other hand, comparing the centercenter positions of the inner and outer sideband doublets with $\Omega$, the positions of the individual atoms relative to standing wave field nodes can be obtained. For the setup in Fig. 2(c), we have $z_{1}=0.05 \lambda, \quad \Omega_{1}=61.80 \gamma$, and $\Omega_{2}$ $=145.79 \gamma$. From the spectrum, using the above procedure, we obtain $\Omega_{1}=(61.58 \pm 6.16) \gamma, \Omega_{2}=(146.22 \pm 14.62) \gamma$, assuming a relative uncertainty of $10 \%$. From $z_{1}$ $=\lambda / 2 \pi \arcsin \left(\Omega_{1} / \Omega\right)$, this would yield a measurement result of $z_{1}=(0.050 \pm 0.005) \lambda$, in good agreement with the actual position of the atoms.
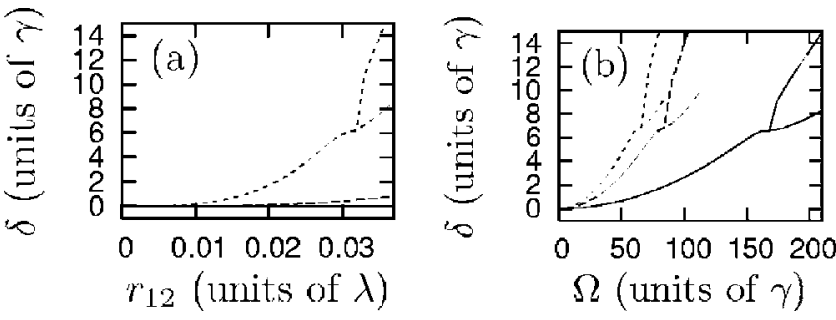

FIG. 4. Deviation $\delta=\nu_{\mathrm{p}}-\Omega_{12}$ of the peak position $\nu_{\mathrm{p}}$ from $\Omega_{12}$ for closely-spaced atoms. $\Delta=0, \theta=\pi / 2$, and (a) against the atomic separation. $z_{1}=0.05 \lambda, \Omega=3 \gamma$ (solid), $20 \gamma$ (dashed), $80 \gamma$ (dotted). (b) Against the driving field Rabi frequency. $z_{12}=0.02 \lambda, z_{1}=0.05 \lambda$ (solid), $0.125 \lambda$ (dashed), $0.2 \lambda$ (dotted). Branches indicate splittings into two peaks.

In the above two regimes, the situation slightly complicates if both atoms are located near-symmetrically around a node or an antinode. In this case, $\Omega_{1} \approx \Omega_{2}$, such that the two sideband peaks (or doublets) overlap. One way to resolve this is to adequately change the standing wave field phase. By this, the symmetry can be lifted to give $\Omega_{1} \neq \Omega_{2}$. Then, the above procedure can be applied to yield the separation and positions.

If the two atoms are very close to each other (distance $\lesssim \lambda / 30$ ), then the spectrum is dominated by the dipole-dipole interaction energy $\Omega_{12}$, which gives rise to sideband structure at each side of the fluorescence spectrum close to $\omega_{L} \pm \Omega_{12}$, and only weakly depends on the driving field. A typical spectrum for this parameter range is shown in Fig. 2(d). As long as $\Omega_{1}, \Omega_{2}, \gamma \ll \Omega_{12}$ is satisfied, the sideband structures only have a small residual dependence on the Rabi frequency. Thus, the sideband peak position $\nu_{\mathrm{p}}$ can directly be identified with $\Omega_{12}$. Figure 4(a) shows the deviation of the sideband peak positions from $\Omega_{12}$ versus the atomic separation distance for different Rabi frequencies $\Omega$. Note that the effective Rabi frequencies $\Omega_{1}, \Omega_{2}$ also depend on the position of the first atom within the wavelength, with maximum values $\Omega_{1}, \Omega_{2} \approx \Omega$ close to the antinodes. It can be seen that for weak $\Omega_{1}, \Omega_{2}$, the experimentally accessible sideband peak position and $\Omega_{12}$ coincide very well. With increasing Rabi frequency, the deviation increases, until the driving field induces a splitting of the sideband peaks, indicated by the branching point in Fig. 4(a). If the initial spectrum of the first measurement has insufficient signal-to-noise ratio, then the fluorescence intensity can be enhanced by increasing the driving field intensity. Note that due to the dependence of $\Omega_{1}, \Omega_{2}$ on the position of the two atoms, different positions of the two atoms may require different laser field intensities. It is also possible to extrapolate the result of several measurements to the driving field-free limit to increase the measurement accuracy. Via Eqs. (2) or (4), the measured $\Omega_{12}$ can easily be used to obtain the interatomic separation. The separation is measured with increasing accuracy in the region of large slope of $\Omega_{12}$ versus $z_{12}$. For maximum accuracy, Eq. (2) should be numerically solved for the separation. Here, we discuss the small separation limit Eq. (4), and allow for a small uncertainty in $\Omega_{12}\left(\Omega_{12} \rightarrow \Omega_{12}+\delta \Omega_{12}\right)$. We obtain $z_{i j}$ $=\left[3 \gamma /\left(2 k^{3} \Omega_{12}\right)\right]^{1 / 3}\left[1-\delta \Omega_{12} /\left(3 \Omega_{12}\right)\right]$ as the distance $z_{i j}$ between the two atoms. Thus, the relative uncertainty of the 
final result is about one-third of the relative uncertainty of the measured $\Omega_{12}$. Consider, for example, the case shown in Fig. 2(d). The actual distance is $z_{12}=0.03 \lambda$. The measured dipole-dipole energy is $\Omega_{12}=(220.5 \pm 22) \gamma$, again with a relative measurement uncertainty of about $10 \%$. From Eq. (2), the distance then evaluates to $z_{12}=(0.030 \pm 0.001) \lambda$. Thus, in this case, the uncertainty of the distance measurement would be about $\lambda / 1000$, i.e., less than $4 \%$ of the actual distance.

Once the distance $z_{12}$ is known, the positions of the two atoms relative to nodes of the standing wave field can be obtained. For this, we note from Fig. 4(b) that-for otherwise fixed parameters - the position of the branching point depends on the Rabi frequencies $\Omega_{1}$, and thus on the position $z_{1}$. If in the experiment we increase $\Omega$ up to the branching point, then the position of the atom pair relative to the field nodes can be deduced. Accurate analytic expressions for the position of the branching point, however, are involved, as the general expression of the fluorescence spectrum is complicated [11]. Thus, a numerical fit as shown in Fig. 4(b) should be used to evaluate $z_{1}$. Finally, since for small distances, the spectrum is almost independent to the driving field, the distance information can also be obtained using a travellingwave field, which may be more convenient in practice.

The precise positioning of the atoms is limited by thermal or quantum position uncertainties [15]. We have simulated this effect by assuming a motional ground-state harmonic oscillation with amplitude $\delta z_{12}=0.005 \lambda$ (corresponding to a Lamb-Dicke parameter $\eta \approx 0.016$ ) around the mean distance $z_{12}$. Results averaged over this motion are shown with dashed lines in Fig. 2. For $\delta z_{12} \ll z_{12}$, the motion is negligible. With increasing ratio $\delta z_{12} / z_{12}$, the spectral peaks split up. In Fig. 2(d), two peaks emerge at the classical turning points of the distance oscillation. From these, the mean distance can again be obtained. The possible separation measurement range is limited, as the dipole-dipole coupling $\Omega_{12}$ increases with decreasing separation as $z_{12}^{-3}$. For our model to remain valid, however, $\Omega_{12} \ll \omega_{0}$ should be fulfilled. From Eq. (4), for $\gamma$ $\sim 10^{7} \mathrm{~Hz}, \Omega_{12} \leqslant 10^{13} \mathrm{~Hz}$, we estimate $z_{12} \geqslant \lambda / 550$ as the theoretical resolution limit. This limitation only applies to the distance of the two atoms itself; the distance uncertainty in principle can be well below $\lambda / 550$. Note that these considerations neglect experimental uncertainties, and are subject to imperfections, e.g., in the measurement of laser field parameters or the alignment of dipole moments or laser fields.

In summary, we have discussed a microscopy scheme entirely based on optical far-field techniques. It allows to measure the separation between and the position of two nearby atoms in a standing wave laser field with fractionalwavelength precision over the full range of distances from about $\lambda / 550$ up to the Rayleigh limit $\lambda / 2$.

This research is supported by the Air Force Office of Scientific Research, DARPA-QuIST, Office of Naval Research, and the TAMU Telecommunication and Informatics Task Force (TITF) initiative.
[1] K. S. Johnson et al., Science 280, 1583 (1998); V. Westphal and S. W. Hell, Phys. Rev. Lett. 94, 143903 (2005).

[2] A. Lewis et al., Ultramicroscopy 13, 227 (1984); A. Lewis et al., Nat. Biotechnol. 21, 1378 (2003).

[3] S. W. Hell, Nat. Biotechnol. 21, 1347 (2003).

[4] U. W. Rathe and M. O. Scully, Lett. Math. Phys. 34, 297 (1995); A. N. Boto et al., Phys. Rev. Lett. 85, 2733 (2000); M. D’Angelo, M. V. Chekhova, and Y. Shih, ibid. 87, 013602 (2001).

[5] M. O. Scully and K. Drühl, Phys. Rev. A 25, 2208 (1982); M. O. Scully and C. H. Raymond Ooi, J. Opt. B: Quantum Semiclassical Opt. 6, S816 (2004); A. Muthukrishnan, M. O. Scully, and M. S. Zubairy, ibid. 6, S575 (2004).

[6] W. Denk, J. H. Strickler, and W. W. Webb, Science 248, 73 (1990).

[7] E. Betzig, Opt. Lett. 20, 237 (1995).

[8] C. Hettich et al., Science 298, 385 (2002).
[9] R. H. Dicke, Phys. Rev. 93, 99 (1954).

[10] Z. Ficek and S. Swain, Quantum Interference and Coherence (Springer, Berlin, 2005).

[11] T. G. Rudolph, Z. Ficek, and B. J. Dalton, Phys. Rev. A 52, 636 (1995); J. G. Cordes and W. Roberts, ibid. 29, 3437 (1984).

[12] A. M. Herkommer, W. P. Schleich, and M. S. Zubairy, J. Mod. Opt. 44, 2507 (1997); E. Paspalakis and P. L. Knight, Phys. Rev. A 63, 065802 (2001).

[13] S. Qamar, S.-Y. Zhu, and M. S. Zubairy, Phys. Rev. A 61, 063806 (2000); S. Qamar, S.-Y. Zhu, and M. S. Zubairy, Opt. Commun. 176, 409 (2000).

[14] F. Ghafoor, S. Qamar, and M. S. Zubairy, Phys. Rev. A 65, 043819 (2002); M. Sahrai, H. Tajalli, K. T. Kapale, and M. S. Zubairy, ibid. 72, 013820 (2005).

[15] J. Eschner et al., Nature (London) 413, 495 (2001); G. R. Guthörlein et al., Nature (London) 414, 49 (2001). 